\section{Is pre-B cell colony-enhancing factor a potential therapeutic target for RA?}

Pre-B cell colony-enhancing factor (PBEF; also known as visfatin) levels are elevated in the serum and synovial fluid of patients with rheumatoid arthritis (RA). Although upregulation of PBEF has been shown to occur via STAT-3-mediated interleukin (IL)-6 signaling, the role of PBEF in joint inflammation has not yet been determined. Brentano et al. isolated synovial fibroblasts (SFs) from patients with $R A$ to study the role of PBEF in the rheumatoid joint.

SFs produced increased levels of PBEF in response to Toll-like receptor ligands, and cytokines IL-1 $\beta$ and tumor necrosis factor, which are involved in signaling pathways of the innate immune system. In SFs from patients with RA, PBEF regulated the production of proinflammatory cytokine IL-6 and matrix metalloproteinase (MMP) 1 and MMP3, matrixdegrading enzymes linked to the progressive destruction of articular cartilage in joints of patients with RA. PBEF also activated the transcription factors nuclear factor- $\kappa \mathrm{B}$ and activator protein-1, which are involved in the initiation and maintenance of inflammatory responses. Finally, PBEF levels correlated with levels of the inflammation marker $\mathrm{C}$-reactive protein and with the Disease Activity Score in 28 joints in patients with RA.

This study, therefore, identifies PBEF as a marker of the severity of inflammation in patients with RA. The authors hypothesize that PBEF might be a potential therapeutic target for the treatment of this debilitating disease.

Original article Brentano F et al. (2007) Pre-B cell colonyenhancing factor/visfatin, a new marker of inflammation in rheumatoid arthritis with proinflammatory and matrixdegrading activities. Arthritis Rheum 56: 2829-2839

\section{Digital X-ray radiogrammetry can provide outcome measures throughout the course of RA}

Hand bone measurements that show periarticular bone loss have previously been proposed as outcome measurements in patients with early rheumatoid arthritis (RA). Since joint inflammation might occur throughout the entire course of disease, Hoff et al. undertook a longitudinal study to compare dual-energy $\mathrm{X}$-ray absorptiometry (DXA) and digital X-ray radiogrammetry (DXR) measurements of hand bone mineral density (BMD) as potential outcome measures in patients with prolonged disease.

In this study, DXR and DXA BMD measurements were taken for both hands in 215 patients with RA, and were analyzed as average values to avoid bias regarding the dominant and nondominant hands. The participants had mean disease duration of 9 years at the start of the study and were analyzed at two consultations separated by a period of 2 years.

Although DXA measurements only showed significant hand bone loss for those patients who started the study with a disease duration of $\leq 3$ years, DXR measurements showed significant loss in hand BMD regardless of the patient's disease duration. At the start of the study, hand BMD was significantly lower in patients with high disease activity than in other patients, when measured by either DXR or DXA. Hand bone loss also correlated with disease activity when measured by DXR, but not by DXA; patients with high disease activity had greater loss than those with lower activity.

The authors conclude that although hand DXA BMD measurements can only be used as outcome measures for early RA, DXR BMD might be used to provide outcome measures throughout the entire course of disease.

Original article Hoff $\mathrm{M}$ et al. (2007) Hand bone loss as an outcome measure in established rheumatoid arthritis: 2-year observational study comparing cortical and total bone loss. Arthritis Res Ther [doi:10.1186/ar2280]

\section{Apoptotic cell-B cell interactions and IL-10 involved in T-cell-mediated immunoregulation}

Immune tolerance of apoptotic cells (ACs) is essential for the prevention of autoimmunity. Dendritic-cell (DC) uptake of ACs is known to inhibit the DC maturation required for DCmediated activation of naive $T$ cells. This inhibition is overcome by exposure to immunostimulatory events such as immunization or infection. Since B cells also exert antigenpresenting activities, Gray and co-workers have studied the effect of ACs on B-cell-mediated regulation of autoimmune responses.

In vitro and in vivo experiments demonstrated that direct interaction of ACs with activated 